\title{
Radiative decays: a new flavour filter
}

\author{
F.E. Close" \\ Department of Theoretical Physics, University of Oxford, \\ Keble Rd., Oxford, OX1 3NP, United Kingdom \\ A. Donnachie' \\ Department of Physics and Astronomy, University of Manchester \\ Manchester M13 9PL, United Kingdom \\ Yu.S. Kalashnikovaf \\ ITEP \\ Moscow, Russia
}

\begin{abstract}
Radiative decays of the $1^{3} D_{1}$ orbital excitations of the $\rho, \omega$ and $\phi$ to the scalars $f_{0}(1370), f_{0}(1500)$ and $f_{0}(1710)$ are shown to provide a flavour filter, clarifying the extent of glueball mixing in the scalar states. A complementary approach to the latter is provided by the radiative decays of the scalar mesons to the ground-state vectors $\rho, \omega$ and $\phi$. Discrimination among different mixing scenarios is strong.
\end{abstract}

\footnotetext{
${ }^{1}$ e-mail: f.close@physics.ox.ac.uk

${ }^{2}$ e-mail: ad@a35.ph.man.ac.uk

${ }^{3}$ e-mail: yulia@heron.itep.ru
} 


\section{Introduction}

The observation and confirmation of gluonic degrees of freedom in mesonic states is of great significance for QCD, which predicts the existence of glueballs (bound states of gluons) and of hybrids (quark-antiquark-gluon states). Calculations in lattice QCD give estimates of the likely masses of glueballs [1] and light-quark hybrids [2], and evidence for the excitation of gluonic degrees of freedom has emerged in several processes. Lattice calculations predict that the lightest glueball has $J^{P C}=0^{++}$ and is in the mass range 1.3 to $1.7 \mathrm{GeV}$. Experimentally [3] there is one more $0^{++}$state in this mass range than can be accomodated by excited quark-antiquark states. The natural inference is that there is a glueball state present [4, 5]. A clear exotic resonance, $\pi_{1}(1600)$, with quantum numbers $J^{P C}=1^{-+}$which cannot be accessed by a pure quark-antiquark state, has been seen [6] in the $\eta^{\prime}(958) \pi$ channel in the reaction $\pi^{-} N \rightarrow\left(\eta^{\prime}(958) \pi\right) N$ and in the $\rho^{0} \pi^{-}$channel $\| 7$, 8] in the reaction $\pi^{-} N \rightarrow\left(\pi^{+} \pi^{-} \pi^{-}\right) N$. Anomalously large hadronic decay modes of light-quark vector mesons, that is decays which are predicted to be very small in standard models [9], are observed in $e^{+} e^{-}$annihilation around 1.6 GeV. A favoured explanation [10, 11, 12] is to include vector hybrids which, on the basis of the $\pi_{1}(1600)$ mass, are expected to be in this mass region.

Apart from states with exotic quantum numbers, disentangling hybrids and glueballs from quark-antiquark states is difficult using only hadronic decay channels because of mixing. However, radiative transitions offer special opportunities. The coupling to the charges and spins of constituents reveals detailed information about wave functions and discriminates among models. In the case of gluonic excitations of the $\pi$ and $\rho$, that is hybrids, the spin structure differs from conventional excitations with the same overall $J^{P C}$. For example, in a hybrid $1^{--}$the $q \bar{q}$ are in a spin-singlet, while for the $0^{-+}$they are in a spin-triplet: in each case this is the reverse of what one is accustomed to. In the case of the scalar mesons a direct measure of their electromagnetic couplings gives information about the flavour content of the scalar states and could resolve the issue of $G-q \bar{q}$ mixing.

We have calculated [13] the rates for the radiative decays $V^{*} \rightarrow \gamma M$, where the $V^{*}$ are the $2 S$ and $1 D$ excitations of the $\rho, \omega$ and $\phi$ in the 1.4 to $1.8 \mathrm{GeV}$ mass range and the $M$ are positive C-parity $q \bar{q}$ states in the 1.2 to $1.7 \mathrm{GeV}$ mass range. We label the vector states $\rho_{S}, \rho_{D}, \omega_{S}, \omega_{D}, \phi_{S}$ and $\phi_{D}$. Some of these vector decays are predicted to have branching ratios in excess of $10^{-2}$ and will be measurable at existing and planned facilities, such as $e^{+} e^{-}$annihilation by Initial State Radiation (ISR) at Babar and Belle, by direct $e^{+} e^{-}$annihilation at the upgraded VEPP collider at Novosibirsk and by diffractive photoproduction following the upgrade at Jefferson Laboratory.

These radiative transitions serve two purposes, exploring the nature both of the 
initial excited vector state and that of the resultant meson. In $[13]$ we concentrated on the former aspect. Here we emphasise the latter and extend the calculation to the radiative decays of the scalars to the ground-state vector mesons. There is again great potential for precise measurement of these decays, for example at CLEO via the decay $J / \psi \rightarrow \gamma \gamma V$ or at COMPASS via central production in high-energy proton proton collisions.

Radiative transitions of $\rho^{*}$ or $\omega^{*}$ to $\gamma M$ couple directly to the $u \bar{u} \pm d \bar{d}$ content of $M$. In similar vein, the analogous transitions involving $\phi^{*}$ couple to the $\beta$ content of $M$. By comparing the relative rates for isovector $\left(|M\rangle \equiv \frac{1}{\sqrt{2}}(|u \bar{u}\rangle-|d \bar{d}\rangle)\right.$ and isoscalar $\left(|M\rangle \equiv \cos \theta|\beta\rangle+\sin \theta \frac{1}{\sqrt{2}}(|u \bar{u}\rangle+|d \bar{d}\rangle)\right)$ for a set of mesons $M$ of positive C-parity and the same $J^{P}$ it is possible in principle to determine the relative amount of $\beta$ and non-strange flavours in the $M$ wavefunction and hence to weigh the flavour content of the nonet. In the case where a glueball has mixed into the multiplet extending it to a decuplet, as is hypothesised 4, 5] to be the case for the $f_{0}(1370), f_{0}(1500)$ and $f_{0}(1710)$, such transitions enable the role of the glueball to be disentangled and might even be able to estabish the mass of the "quenched" glueball [5]. Furthermore, as we shall show, radiative decays of the scalar mesons to the ground-state vectors $\rho(770)$ and $\phi(1020)$ are also sensitive probes of the glueball mixing in the scalars.

\section{The model}

The details of the radiative decay calculation are described in [13]. Wavefunctions for the excited vector mesons are found variationally from the Hamiltonian

$$
H=\frac{p^{2}}{m_{q}}+\sigma r-\frac{4}{3} \frac{\alpha_{s}}{r}+C
$$

with standard quark model parameters $m_{q}=0.33 \mathrm{GeV}$ for $u$ and $d$ quarks and 0.45 $\mathrm{GeV}$ for $s$ quarks, $\sigma=0.18 \mathrm{GeV}^{2}$ and $\alpha_{s}=0.5$. The wavefunctions are taken to be Gaussian of form $\exp \left(-p^{2} /\left(2 \beta_{M}^{2}\right)\right)$ multiplied by the appropriate polynomials and $\beta_{M}$ treated as the variational parameter in $H$ for each of the $1 S, 1 P, 2 S, 1 D$ states. Where data already exist good agreement was found.

For transitions involving excited states with nodes in their wavefunctions there is a priori concern about the stability of the calculations. This does not arise in the transitions we consider here, and the approximation of harmonic oscillator wave functions is 'safe'. This can be seen, for example, in the $1 P \rightarrow 1 S$ transitions. Using the operator relation $\vec{p}=i m_{q}[H \vec{r}] / 2$, where $H$ is the Hamiltonian (1), one obtains for the radial integrals of the wave functions the equality

$$
\langle B|p| A\rangle=\frac{i m_{q}}{2} \omega\langle B|r| A\rangle,
$$


where $A$ is the initial meson, $B$ is the final meson, $\omega=m_{A}-m_{B}$ and $m_{A}$ and $m_{B}$ are the model, not the physical masses. With Gaussian wave functions the radial integrals (up to a phase) are

$$
\langle B|p| A\rangle=\frac{1}{\sqrt{2}} \frac{\beta^{5}}{\beta_{A}^{5 / 2} \beta_{B}^{3 / 2}}
$$

and

$$
\langle B|r| A\rangle=\frac{1}{\sqrt{2}} \frac{\beta_{A}^{5 / 2} \beta_{B}^{3 / 2}}{\beta_{0}^{5}}
$$

where

$$
\beta^{-2}=\frac{1}{2}\left(\beta_{A}^{-2}+\beta_{B}^{-2}\right)
$$

and

$$
\beta_{0}^{2}=\frac{1}{2}\left(\beta_{A}^{2}+\beta_{B}^{2}\right)
$$

From [13], $\beta_{A}=0.274 \mathrm{GeV}, \beta_{B}=0.313 \mathrm{GeV}$ and the mass difference $\omega=0.562$ $\mathrm{GeV}$. So we find

$$
\frac{\langle A|p| B\rangle}{m_{q}}=0.658
$$

and

$$
\frac{\omega\langle A|r| B\rangle}{2}=0.616
$$

The deviation between (7) and (8) is less than 10 percent so, as the squares enter the widths, the uncertainty due to the use of Gaussian wave functions is about 20 percent. This is appreciably less than the effects we are considering.

A similar exercise for $1 D \rightarrow 1 P$ gives

$$
\frac{\langle B|p| A\rangle}{m_{q}}=1.088
$$

and

$$
\frac{\omega\langle B|r| A\rangle}{2}=1.024
$$

so in this case the uncertainty due to the use of Gaussian wave functions is less than 10 percent.

The pure electric-dipole $(E 1)$ transition is well-defined for heavy quarks but is certainly a bad approximation for light quarks. We include the magnetic quadrupole (M2) transition as well and there is a long history of success with this approach, even though the $M 2$ terms are at the same order in $p^{2}$ as $E 1$ corrections proportional to the anomalous magnetic moment of the constituents, spin-orbit terms, Thomas 
precession and binding effects[14, 15]. Some of these corrections can be calculated 14, 15, 16, 17, 18]; some can only be estimated [14]. In any case, going beyond the leading approximation for the electric and magnetic amplitudes requires knowledge of the Lorentz nature of the confinement force.

The most notable successes of this approach, namely of calculating the leading terms for each of the relevant electric and magnetic multipoles, have been in reproducing the magnitudes and relative phases of over 100 helicity amplitudes for photoexcitation of the proton and neutron [19]. These give a clear indication of which amplitudes are large or small, and of their relative sizes and signs. This success suggests that although corrections may be individually significant, their collective effect is small.

It is possible to go beyond such an approximation, but at the price of losing some predictive power. Within the general assumption that electromagnetic amplitudes are additive in the constituents, it is possible to obtain relations among the helicity amplitudes, angular distributions and widths for a set of states by normalising the reduced amplitude to some subset of observables [20, 21].

This is the philosophy that we shall adopt. First, within the "leading multipole" hypothesis we can make two checks of our procedures.

(i) We find that our result for the decay $f_{1}(1285) \rightarrow \gamma \rho$ is in good accord with experiment [3, 22, 23].

(ii) We predict that $\Gamma\left(f_{2}(1270) \rightarrow \gamma \rho\right)<\Gamma\left(f_{1}(1285) \rightarrow \gamma \rho\right)$, which appears also to be in accord with experiment in that there is no evidence for the radiative decay of the $f_{2}(1270)$ in either the MARKIII [22] or WA102 [23, 24] experiments, and both have strong $f_{2}$ signals.

Secondly, within the "single-quark-transition" hypothesis we can form a positivity constraint among a combination of the widths. This is satisfied by our explicit model dependent calculations, but enables us to draw a more general conclusion, namely that $\Gamma\left(f_{0} \rightarrow \gamma \rho\right) \sim \Gamma\left(f_{1} \rightarrow \gamma \rho\right)$.

\section{Vector meson decays}

Our interest here is in the radiative decays of the $\rho_{D}$, which we identify with the $\rho(1700)$ [3], to $f_{0}(1370)$ and $f_{0}(1510)$ and of the (unobserved) $\phi_{D}$, to which we assign a mass of about $1.9 \mathrm{GeV}$, to $f_{0}(1710)$. In the absence of glueball mixing we predict $13 \pi \Gamma\left(\rho_{D} \rightarrow \gamma f_{0}^{n \bar{n}}(1370)\right) \sim 900 \mathrm{keV}, \Gamma\left(\rho_{D} \rightarrow \gamma f_{0}^{n \bar{n}}(1500)\right) \sim 600 \mathrm{keV}$ (assuming that one or the other is a pure $n \bar{n}$ state) and $\Gamma\left(\phi_{D} \rightarrow \gamma f_{0}^{s \bar{s}}(1710)\right) \sim 200 \mathrm{keV}$. These widths can be changed substantially when glueball mixing is included, the degree of modification depending on the mass of the bare glueball.

Three different mixing scenarios have been proposed: the bare glueball is lighter than the bare $n \bar{n}$ state [25]; its mass lies between the bare $n \bar{n}$ state and the bare $s \bar{s}$ state 
[25]; or it is heavier than the bare $s \bar{s}$ state [26]. We denote these three possibilities by $\mathrm{L}, \mathrm{M}$ and $\mathrm{H}$ respectively. In [13] we identified potentially powerful ways of determining glueball mixing in the scalar mesons through the radiative transitions $\rho_{D} \rightarrow \gamma f_{0}(1370)$ and $\gamma f_{0}(1500)$. If the bare glueball is light $(\sim 1300 \mathrm{MeV})$ it will mix strongly with the $f_{0}^{n \bar{n}}(1370)$ and dilute the $900 \mathrm{keV}$ width which will be pushed into the other scalars, in particular into the $f_{0}^{n \bar{n}}(1500)$ reversing the relative magnitudes of the radiative widths. At the other extreme, the mixing of a heavy glueball $(\sim 1700 \mathrm{MeV})$ does not materially affect the radiative width to the $f_{0}(1370)$ but severely depresses that to the $f_{0}(1500)$ to $100 \mathrm{keV}$ or less. Thus the relative widths of $\rho_{D} \rightarrow \gamma f_{0}(1370)$ and $\rho_{D} \rightarrow \gamma f_{0}(1500)$ are sensitive to the glueball mass. There is similar sensitivity in the decays $\phi_{D} \rightarrow \gamma f_{0}(1500)$ and $\phi_{D} \rightarrow \gamma f_{0}(1710)$. For a light glueball the former is essentially zero and the latter is predicted to be $\sim 170$ $\mathrm{keV}$. For a heavy glueball the situation is reversed, with the decay $\phi_{D} \rightarrow \gamma f_{0}(1500)$ predicted to be about $260 \mathrm{keV}$ and the decay $\phi_{D} \rightarrow \gamma f_{0}(1710)$ to be essentially zero. These branching ratios are challenging but important as they open the possibility of weighing the $n \bar{n}$ and $\beta$ flavour content of the scalar states and determining the bare mass of the scalar glueball. The only question is whether experiment will be sensitive to such magnitudes.

In the isovector-scalar sector only the $a_{0}(980)$ is well-established. The mass, and even the existence, of the $a_{0}(1450)$ remains controversial. Its mass is a critical parameter in mass matrices for the mixing of the scalar glueball with the $q \bar{q}$ nonet. We predict $\Gamma\left(\omega_{D} \rightarrow \gamma a_{0}(1450)\right) \sim 610 \mathrm{keV}$ and $\Gamma\left(\rho_{D} \rightarrow \gamma a_{0}(1450)\right) \sim 85 \mathrm{keV}$. Of course in $e^{+} e^{-}$annihilation or diffractive photoproduction the $\rho_{D}$ will be produced at approximately nine times the rate of the $\omega_{D}$, so the $\gamma a_{0}(1450)$ rates will be the same for both. Observation of the $a_{0}(1450)$ in radiative decays would have significant implications, particularly when coupled with the radiative decays to the isoscalar scalars.

Although our emphasis is on the radiative decays to scalars as a probe of their glueball content it is important to look at other decays as a check on the model. We find that the principal radiative decay mode of the $\rho_{D}$ is $\gamma f_{1}(1285)$, with an estimated width [13] of $\sim 1100 \mathrm{keV}$. Although the decays of the $f_{1}(1285)$ are many-bodied, principally $4 \pi$ and $\eta \pi \pi$, this is compensated for by its narrow width of $24 \mathrm{MeV}$. The largest radiative decay of the $\omega_{D}$ is found to be $\gamma a_{1}(1260)$, with a width $\sim 1100 \mathrm{keV}$. Observation of these modes would confirm our calculations. The E852 experiment at Brookhaven has observed [27] the decay $\omega(1640) \rightarrow \omega \eta$. If the $\omega(1640) \equiv \omega_{D}$ then, in the ${ }^{3} P_{0}$ model, the partial width for this mode is predicted [9] to be $13 \mathrm{MeV}$. Thus, if the ${ }^{3} P_{0}$ model is a good guide in this case, a measureable $\omega(1640) \rightarrow \gamma a_{1}$ is anticipated: as E852 have several thousand events in the $\omega \eta$ channel, it is possible that there could be several hundred $\gamma a_{1}$ events in their experiment. 


\section{Scalar meson decays}

Just as the radiative decays of excited vector states to the scalars provide sensitive tests of their parton content, so do the radiative decays of the scalars to the groundstate vectors. In our model these decays are given by

$$
\Gamma\left(f_{0} \rightarrow \gamma V\right)=\frac{8}{3} \alpha p \frac{E_{B}}{m_{A}} \frac{\beta^{2}}{m_{q}^{2}} F^{2}\left(1+\lambda \frac{p^{2}}{\beta^{2}}\right)^{2} I
$$

where $A$ refers to the initial scalar meson, $B$ to the final vector $(V)$ meson,

$$
\begin{gathered}
\lambda=\frac{\beta_{A}^{2}}{2\left(\beta_{A}^{2}+\beta_{B}^{2}\right)} \\
F=\frac{\beta^{4}}{\beta_{A}^{5 / 2} \beta_{B}^{3 / 2}} \exp \left(-\frac{p^{2}}{8\left(\beta_{A}^{2}+\beta_{B}^{2}\right)}\right)
\end{gathered}
$$

where $\beta$ is given in (5) and $I$ is an isospin factor, $\frac{1}{4}$ for $n \bar{n} \rightarrow \gamma \rho$ and $\frac{1}{9}$ for $s \bar{s} \rightarrow \gamma \phi$. From [13], $\beta_{A}=0.274 \mathrm{GeV}, \beta_{B}=0.313 \mathrm{GeV}$ for $n \bar{n}$ states and $\beta_{A}=0.307$ $\mathrm{GeV}, \beta_{B}=0.355 \mathrm{GeV}$ for $s \bar{s}$ states. In the absence of glueball mixing it gives $\Gamma\left(f_{0}(1370) \rightarrow \gamma \rho\right) \sim 2300 \mathrm{keV}$, and $\Gamma\left(f_{0}(1710) \rightarrow \gamma \phi\right) \sim 870 \mathrm{keV}$

The width of the decay $f_{1}(1285) \rightarrow \gamma \rho$ is measured and provides a check on the model. This decay is given by

$$
\Gamma\left(f_{1} \rightarrow \gamma V\right)=\frac{8}{3} \alpha p \frac{E_{B}}{m_{A}} \frac{\beta^{2}}{m_{q}^{2}} F^{2}\left(1+\lambda \frac{p^{2}}{\beta^{2}}+\frac{1}{2} \lambda^{2} \frac{p^{4}}{\beta^{4}}\right) I
$$

which for an $n \bar{n}$ state predicts a width of $1400 \mathrm{keV}$. This compares well with the experimental value [3, 22, 23] of $1320 \pm 312 \mathrm{keV}$.

The decay $f_{2}(1270) \rightarrow \gamma \rho$ is given by

$$
\Gamma\left(f_{2} \rightarrow \gamma V\right)=\frac{8}{3} \alpha p \frac{E_{B}}{m_{A}} \frac{\beta^{2}}{m_{q}^{2}} F^{2}\left(1-\lambda \frac{p^{2}}{\beta^{2}}+\frac{7}{10} \lambda^{2} \frac{p^{4}}{\beta^{4}}\right) I
$$

which is rather smaller than the others, due to the negative contribution of the $p^{2} / \beta^{2}$ term. This predicts a width of $644 \mathrm{keV}$.

Experimentally this width is small as neither the MARKIII [22] nor the WA102 23] experiments has any evidence for it. The branching fractions for the radiative decay of $J / \psi$ to $f_{1}(1285)$ and $f_{2}(1270)$ are comparable [3], at $(6.1 \pm 0.9) \times 10^{-4}$ and $(1.39 \pm 0.14) \times 10^{-3}$ respectively, so the non-observation of any $f_{2}(1270)$ signal in the

decay $J / \psi \rightarrow \gamma(\gamma \rho)$ is meaningful. A similar situation holds in central production in 
high-energy proton-proton interactions 23] and one can deduce [24] an upper limit on $\Gamma\left(f_{2} \rightarrow \gamma \rho\right)$ of $500 \mathrm{keV}$ at $95 \%$ confidence level. So it is reasonable to suppose that our results for the $f_{0}$ radiative decays are valid.

As in 13] we consider three scenarios for the glueball mass: light, medium and heavy. The predicted widths for the decays of $f_{0}(1370), f_{0}(1500)$ and $f_{0}(1710)$ to $\gamma \rho$ and $\gamma \phi$ are given in table 1 .

\begin{tabular}{|c|ccc|ccc|}
\hline & \multicolumn{3}{|c|}{$\rho(770)$} & \multicolumn{3}{c|}{$\phi(1020)$} \\
& $\mathrm{L}$ & $\mathrm{M}$ & $\mathrm{H}$ & $\mathrm{L}$ & $\mathrm{M}$ & $\mathrm{H}$ \\
\hline$f_{0}(1370)$ & 443 & 1121 & 1540 & 8 & 9 & 32 \\
\hline$f_{0}(1500)$ & 2519 & 1458 & 476 & 9 & 60 & 454 \\
\hline$f_{0}(1710)$ & 42 & 94 & 705 & 800 & 718 & 78 \\
\hline
\end{tabular}

Table 1. Effect of mixing in the scalar sector of the $1^{3} P_{0}$ nonet for radiative decays to $\rho$ and $\phi$. The radiative widths, in $\mathrm{keV}$, are given for three different mixing scenarios as described in the text: light glueball (L), medium-weight glueball (M) and heavy glueball $(\mathrm{H})$.

It is clear from the table that the discrimination among the different mixing scenarios is strong. The decay $f_{0}(1500) \rightarrow \gamma \rho$ is perhaps the most interesting because of its comparatively narrow total width of $\sim 120 \mathrm{MeV}$. This enhances the radiative decay branching fraction, which is $\sim 2 \%$ for a light glueball, $\sim 1 \%$ for a medium glueball and $\sim 0.5 \%$ for a heavy glueball. The absolute magnitudes may vary by $\sim 20 \%$ due to the uncertainties in the model, but the relative strengths manifested in the pattern above should be robust. Even allowing for the intrinsic uncertainties, there appears to be a solid conclusion that these rates are worth pursuing experimentally. We now assess further the robustness of these results by looking at the more general structures that follow from the single-quark-transition property of the dynamics.

\section{Single quark transition}

The results above depend upon the rather general assumption that electromagnetic amplitudes are additive in the constituents. Independent of details of binding dynamics, it is possible to obtain relations among the helicity amplitudes, and hence angular distributions and widths, that depend only on the assumption that the mesons are described as $q \bar{q}$ states in the $P$ and $S$ states respectively.

For example, in the electric-dipole approximation, the relative widths for transitions from $f_{J} \rightarrow \gamma \rho$ would be equal, apart from phase space corrections. Given the magnitude of one as input, the others immediately follow. While such results are a 
good approximation for heavy flavours [3], for the lighter $u, d, s$ flavours, significant magnetic-quadrupole may be expected, as is confirmed by the explicit calculations above. (Electric octupole contributions, while allowed in general for $f_{2} \rightarrow \gamma V$, will vanish if, as assumed here, the vector $V$ is a pure ${ }^{3} S_{1}$ state, and the $f_{2}$ is a pure ${ }^{3} P_{2}$ state [20].)

Within this assumption that the electromagnetic transition amplitude is additive in the constituents [20, 21] and that the relevant $q \bar{q}$ states can be classified as $S, P$ levels, sum rules can be obtained. We can of course check that these sum rules are satisfied in particular explicit models.

The most general decomposition of the helicity amplitudes for ${ }^{3} S_{1} \leftrightarrow{ }^{3} P_{J}$ radiative transitions into electric-dipole and magnetic-quadrupole terms is given in [17, 18]. The helicity amplitudes may be written as:

$$
\begin{aligned}
& { }^{3} S_{1} \leftrightarrow{ }^{3} P_{0} \\
& A_{0}=\sqrt{2}\left(E_{1}+2 E_{R}\right) \\
& { }^{3} S_{1} \leftrightarrow{ }^{3} P_{1} \\
& A_{0}=\sqrt{3}\left(E_{1}+E_{R}+M\right) A_{1}=\sqrt{3}\left(E_{1}+E_{R}-M\right) \\
& { }^{3} S_{1} \leftrightarrow{ }^{3} P_{2} \\
& A_{0}=\left(E_{1}-E_{R}+3 M\right) \quad A_{1}=\sqrt{3}\left(E_{1}-E_{R}+M\right) \quad A_{2}=\sqrt{6}\left(E_{1}-E_{R}-M\right)
\end{aligned}
$$

Here $E_{1}$ is the leading electric-dipole term, $E_{R}$ is the "extra" electric-dipole term and $\mathrm{M}$ is the magnetic-quadrupole term. In terms of our model, $E_{1}$ comes from the convection current and $E_{R}$ and $M$ from the spin-flip one (see equation (2) of [13]). The width for the decays $V \rightarrow \gamma f_{J}$ is

$$
\Gamma\left(V \rightarrow \gamma f_{J}\right)=\frac{p^{3}}{3} \sum_{\lambda}\left|A_{\lambda}\right|^{2}
$$

and for the decays $f_{J} \rightarrow \gamma V$ is

$$
\Gamma\left(f_{J} \rightarrow \gamma V\right)=\frac{p^{3}}{2 J+1} \sum_{\lambda}\left|A_{\lambda}\right|^{2}
$$

Consider first the decays $V \rightarrow \gamma f_{J}$. For equal phase space and equal form factors, we can eliminate the $|M|^{2}$ term by the combination $3 \Gamma\left(V \rightarrow \gamma f_{1}\right)-\Gamma\left(V \rightarrow \gamma f_{2}\right)$. This leaves cross terms between $E_{1}$ and $E_{R}$ which are in general of indeterminate sign. However, these too can be eliminated by including $\Gamma\left(V \rightarrow \gamma f_{0}\right)$ and a combination formed that is proportional to the sum $\left|E_{1}\right|^{2}+\left|E_{R}\right|^{2} \geq 0$.

Thus, for equal phase space and equal form-factors, one would have

$$
\Gamma\left(\rho(2 S) \rightarrow \gamma f_{2}\right)+7 \Gamma\left(\rho(2 S) \rightarrow \gamma f_{0}\right) \geq 3 \Gamma\left(\rho(2 S) \rightarrow \gamma f_{1}\right)
$$


Similarly for the decays of pure $n \bar{n} f_{J}$ states,

$$
5 \Gamma\left(f_{2} \rightarrow \gamma \rho\right)+7 \Gamma\left(f_{0} \rightarrow \gamma \rho\right) \geq 9 \Gamma\left(f_{1} \rightarrow \gamma \rho\right)
$$

It is straightforward to verify that (21) is satisfied by equations (17) to (19) ff of [13] and that (22) is satisfied by (11), (14) and (15) of the present paper.

Given that empirically $\Gamma\left(f_{1} \rightarrow \gamma \rho\right) \sim 1500 \mathrm{keV}$, equation (22) implies that one or other of the $f_{2}$ or $f_{0}$ must have a radiative width of at least $\sim 1000 \mathrm{keV}$. To the extent that there is no clear sign of the $f_{2} \rightarrow \gamma \rho$ decay in either the data sets of 22, 23. one may anticipate that the $f_{0} \rightarrow \gamma \rho$ could be large, in line with our specific calculations. In any event, such states should be sought in $\psi \rightarrow \gamma \gamma \rho$.

\section{Conclusions}

Our computations show that radiative decay rates can be large for some transitions. The limitation for detection of these processes seems to be one primarily of acceptance. These processes should be borne in mind when detectors for future experiments, for example at Hall D in Jefferson Lab, are being designed. We advocate that existing data be mined to seek evidence of radiative decays, for example in E852 where the decay $\omega(1640) \rightarrow \gamma a_{1}$ should be present at a reasonable rate. If radiative transitions are observed, then the use of radiative decays will be proven as a viable technique.

It is important to compare photoproduction and $e^{+} e^{-}$annihilation as these give complementary information on the vector-meson wavefunctions. Jefferson Laboratory is an obvious place for the photoproduction studies, and as regards $e^{+} e^{-}$, VEPP is the immediate natural candidate, though there is uncertainty as to whether its luminosity will be enough. DAFNE operating up to its maximum energy promises high luminosity in the future. Initial state radiation at BaBar, Belle and CLEO-c could give significant rates for $e^{+} e^{-}$annihilation at $\sqrt{s} \leq 2 \mathrm{GeV}$.

A complementary approach to the role of glue in the scalar wave functions is provided by the decays of the scalars to $\gamma \rho$ and $\gamma \phi$. Both the $f_{0}(1500)$ and $f_{0}(1710)$ have been clearly observed in the radiative decays $J / \psi \rightarrow \gamma X$, and we recommend that a detailed exploration of the scalar states should be planned as part of the CLEO-c programme.

Radiative transitions provide a new flavour filter that can clarify the nature of the scalar mesons in the 1.2-1.8 GeV region, in particular the role of glue in the scalar wavefunctions.

\footnotetext{
${ }^{4}$ There is a sign error in equation (18) in $\left[13\right.$. The sign of the $G_{E} G_{M}$ term should be negative, as it is in equation (A5).
} 


\section{Acknowledgements}

This work is supported, in part, by grants from the Particle Physics and Astronomy Research Council, RFBR 00-15-96786, INTAS-RFBR 97-232 and the EU-TMR program "Eurodafne", NCT98-0169.

\section{References}

[1] SESAM Collaboration, G. Bali et al., Nucl.Phys.Proc.Suppl. 63, 209 (1997); IBM Collaboration, J. Sexton et al., Phys.Rev.Lett. 75, 4563 (1995); F.E. Close and M.J. Teper, Report no. RAL-96-040/OUTP-96-35P; C.J. Morningstar and M. Peardon, Phys.Rev. D 56, 4043 (1997); D. Weingarten, Nucl.Phys.Proc.Suppl. 53, 232 (1997), ibid 63, 194 (1998), ibid 73, 249 (1999); C. McNeile and C. Michael, hep-lat/0010019

[2] P. Lacock, C. Michael, P. Boyle and P. Rowland, Phys.Lett. B 401, 308 (1997); C. Bernard et al., Phys.Rev. D 56, 7039 (1997) and hep-lat/9809087; P. Lacock and K. Schilling, hep-lat/9809022; C. McNeile, hep-lat/9904013; C. Morningstar, hep-ph/0009314

[3] Particle Data Group, Eur.Phys.J. C 15, 1 (2000)

[4] C. Amsler and F.E. Close, Phys. Lett. B 353, 385 (1995)

[5] F.E. Close and A. Kirk, Z.Phys. C 21, 531 (2001)

[6] E852 Collaboration, I.I. Ivanov et al., Phys.Rev.Lett. 86, 3977 (2001)

[7] E852 Collaboration, D.P. Weygand, in HADRON'97, edited by S-U Chung and H.J. Willutski (American Institute of Physics, New York, 1998), p.313

[8] VES Collaboration, Yu.P. Gouz, in Proceedings of XXVI ICHEP, Dallas, (1992), edited by J.R. Sanford, p.572

[9] T. Barnes, F.E. Close, P.R. Page and E.S. Swanson, Phys.Rev. D 55, 4157 (1997)

[10] A. Donnachie and Yu.S. Kalashnikova, Z.Phys. C 59, 621 (1993)

[11] F.E. Close and P.R. Page, Phys.Rev. D 56, 1584 (1997)

[12] A. Donnachie and Yu.S. Kalashnikova, Phys.Rev. D 60, 114011 (1999) 
[13] F.E. Close, A. Donnachie and Yu.S. Kalashnikova, Phys.Rev. D 65, 092003 (2002)

[14] A. Le Yaouanc, L. Oliver, O. Pene and J.C. Raynal, Z.Phys. C 4077 (1988)

[15] F.E. Close and H. Osborn, Phys.Lett. B 34400 (1971); G. Hardekopf and J. Sucher, Phys.Rev. D 252938 (1982); R. McClary and N. Byers, Phys.Rev. D 281692 (1983)

[16] F. Foster and G. Hughes, Z.Phys C 14123 (1982); F.E. Close and Z.P. Li, Phys.Rev. D 422194 (1990)

[17] F.J. Gilman and I. Karliner, Phys.Rev. D 10, 2194 (1974)

[18] G. Karl, S. Meshkov and J. Rosner, Phys.Rev.Lett. 45, 215 (1980); S. Godfrey, G. Karl and P. O'Donnell, Z.Phys C 31, 77 (1986)

[19] L.A. Copley, G. Karl and E. Obryk, Nucl.Phys. B 13, 303 (1969); R.P. Feynman, M. Kislinger and F. Ravndal, Phys.Rev. D 3, 2706 (1971)

[20] F.E. Close, Introduction to Quarks and Partons, (Academic Press), chapter 7

[21] H.J. Melosh, Phys.Rev. D 9, 1095, (1974); F.E. Close, H. Osborn and A.M. Thomson, Nucl. Phys. B 77, 281 (1974); A.J.G. Hey and J. Weyers, Phys.Lett. B 46, 69 (1974); J. Babcock and J. Rosner, Phys.Rev. D 14, 1286 (1976)

[22] D. Coffman et al (MarkIII Collabroation), Phys.Rev. D 41, 1410 (1990)

[23] D. Barberis et al (WA102 Collaboration), Phys.Lett. B 440, 225 (1998)

[24] A. Kirk, private communication

[25] F.E. Close and A. Kirk, Eur.Phys.J. C 21, 531 (2001)

[26] W. Lee and D. Weingarten, Phys.Rev. D 61, 014015 (2000)

[27] E852 Collaboration, P. Eugenio et al., Phys.Lett. B 497, 190 (2001) 